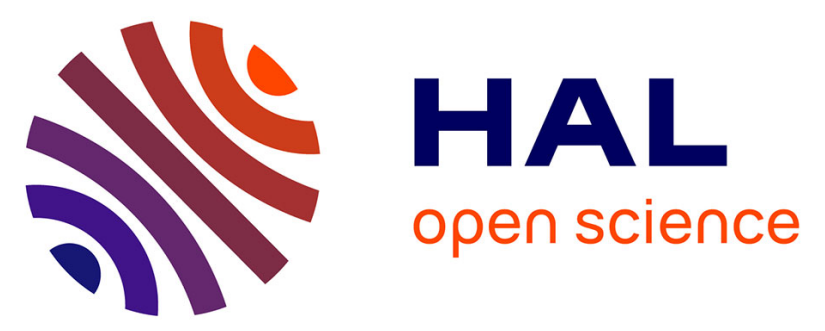

\title{
Apport de la TEP-FDG dans le staging initial des cancers du sein localement avancés traités par chimiothérapie néo-adjuvante
}

Pacôme Fosse, Sylvie Girault, Olivier Capitain, Isabelle Valo, Francis Bouchet, Laurent Vervueren, Franck Lacœuille, Olivier-François Couturier, Olivier Morel

\section{To cite this version:}

Pacôme Fosse, Sylvie Girault, Olivier Capitain, Isabelle Valo, Francis Bouchet, et al.. Apport de la TEP-FDG dans le staging initial des cancers du sein localement avancés traités par chimiothérapie néo-adjuvante. Médecine Nucléaire - Imagerie Fonctionnelle et Métabolique, 2012, 36 (2), Non spécifié. 10.1016/j.mednuc.2011.11.011 . hal-03179503

\section{HAL Id: hal-03179503 \\ https://univ-angers.hal.science/hal-03179503}

Submitted on 24 Mar 2021

HAL is a multi-disciplinary open access archive for the deposit and dissemination of scientific research documents, whether they are published or not. The documents may come from teaching and research institutions in France or abroad, or from public or private research centers.
L'archive ouverte pluridisciplinaire HAL, est destinée au dépôt et à la diffusion de documents scientifiques de niveau recherche, publiés ou non, émanant des établissements d'enseignement et de recherche français ou étrangers, des laboratoires publics ou privés. 


\title{
Apport de la TEP-FDG dans le staging initial des cancers du sein localement avancés traités par chimiothérapie néo-adjuvante
}

\section{FDG-PET in the initial staging of locally advanced breast cancer before neoadjuvant chemotherapy}

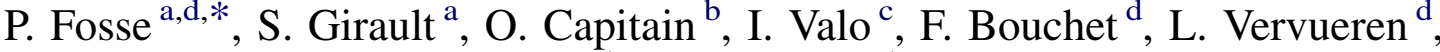 \\ F. Lacoeuille ${ }^{\mathrm{d}}$, O. Couturier ${ }^{\mathrm{d}}$, O. Morel $^{\mathrm{a}}$ \\ ${ }^{a}$ Service de médecine nucléaire, ICO Paul-Papin, 2, rue Moll, 49933 Angers cedex 9, France \\ ${ }^{\mathrm{b}}$ Service d'oncologie médicale, ICO Paul-Papin, 2, rue Moll, 49933 Angers cedex 9, France \\ ${ }^{\mathrm{c}}$ Service d'anatomopathologie, ICO Paul-Papin, 2, rue Moll, 49933 Angers cedex 9, France \\ ${ }^{\mathrm{d}}$ Service de médecine nucléaire, CHU d'Angers, 4, rue Larrey, 49933 Angers cedex 9, France \\ Reçu le 12 septembre 2011 ; accepté le 29 novembre 2011 \\ Disponible sur Internet le 30 janvier 2012
}

\begin{abstract}
Résumé
L'atteinte ganglionnaire axillaire et la présence de métastases à distance sont des facteurs pronostiques majeurs dans la prise en charge du cancer du sein. L'objectif de notre travail a été d'évaluer les performances de la TEP-FDG dans le bilan initial de cancers du sein localement avancés (CSLA) traités par chimiothérapie néo-adjuvante (CNA) et chirurgie, et de comparer les données de la TEP préthérapeutique à celles de l'histologie du curage réalisé après CNA (classification de Sataloff). Cette étude rétrospective a concerné 89 patientes porteuses d'un CSLA, explorées avant mise en route de la CNA par une TEP en complément du bilan d'extension standard (BS). Toutes les patientes ont bénéficié après CNA d'une tumorectomie/mastectomie et d'un curage axillaire. Une atteinte axillaire a été retrouvée chez 58 patientes (65\%) par la TEP et 39 patientes (44\%) par le BS. Comparées à l'histologie du curage axillaire post-CNA, les sensibilité et spécificité de la TEP ont été calculées à $80 \%$ et $63 \%$. La TEP a révélé une atteinte ganglionnaire extra-axillaire, non suspectée par le BS, chez 25 patientes (28\%). Des lésions métastatiques méconnues par le BS ont été découvertes au niveau osseux chez deux patientes et pulmonaire chez une patiente. Un cas de faux-positif TEP hépatique a été observé (adénomatose). Cette étude confirme l'intérêt de la TEP dans le staging initial des CSLA, notamment au niveau ganglionnaire extra-axillaire. Au niveau axillaire, une TEP positive suggère fortement une atteinte métastatique ; les cas d'interprétation douteuse incitant à réaliser en complément une cytoponction échoguidée. (C) 2011 Elsevier Masson SAS. Tous droits réservés.
\end{abstract}

Mots clés : Cancer du sein ; TEP-FDG ; Bilan d'extension ; Curage axillaire

\begin{abstract}
Axillary lymph node and distant metastases are unfavourable prognostic factors in breast cancer. The aim of our study was to evaluate the performance of PET-FDG in the staging of locally advanced breast cancer (LABC) treated with neoadjuvant chemotherapy (NAC) and surgery, and to compare the findings of pretherapeutic PET to those of axillary nodal status obtained by surgery after NAC (Sataloff classification). This retrospective study involved 89 patients with LABC explored at presentation by PET in addition to conventional clinical and imaging staging (CS). Breast cancer excision and axillary dissection were performed after NAC. PET and CS found an axillary involvement in 58 patients (65\%) and 39 patients (44\%), respectively. Compared to the histology of post-NAC axillary dissection, PET had sensitivity and specificity of $80 \%$ and $63 \%$, respectively. PET revealed an extra-axillary lymph node involvement, not suspected by the CS, in 25 patients (28\%). Bone and lung metastases, not suspected by the CS were found in two and one patients, respectively. A case of false-positive PET in the liver was observed (adenomatosis). This study confirms the value of PET in the initial staging of LABC, especially in assessing extra-axillary nodal status. In the determination of axillary status, PET has a high positive predictive value, cases of doubtful interpretation suggesting to perform ultrasound-guided fine needle aspiration in addition.
\end{abstract}

(C) 2011 Elsevier Masson SAS. All rights reserved.

Keywords: Breast cancer; FDG-PET; Initial staging; Axillary dissection

\footnotetext{
* Auteur correspondant.

Adresse e-mail : Pacome_fosse@hotmail.fr (P. Fosse).
} 


\section{Introduction}

Le cancer du sein est le premier cancer chez la femme dans l'Union européenne et aux États-Unis. En France, son incidence est estimée à environ 52500 nouveaux cas en 2010, représentant $34 \%$ des nouveaux cas de cancers féminins, soit $15 \%$ des cancers incidents. Le taux de survie globale à cinq ans est estimé à $85 \%$, mais varie considérablement en fonction du stade initial de la maladie : 98,3\%, 83,5\% et 23,3\%, respectivement, pour les stades local, régional et métastatique [1]. Il représente ainsi la première cause de mortalité par cancer chez la femme avec 11500 décès au cours de l'année 2010 (7,8\% de l'ensemble des décès par cancer tous sexes confondus en France) [2].

Le statut ganglionnaire axillaire et l'atteinte métastatique à distance constituent des facteurs pronostiques majeurs [3]. L'examen clinique et les examens paracliniques réalisés de manière systématique lors du bilan d'extension initial de la maladie (radiographie thoracique, échographie hépatique et scintigraphie osseuse) ne permettent souvent pas de statuer sur une éventuelle atteinte ganglionnaire axillaire, pourtant présente dans plus de $40 \%$ des cas. En cas de cancer localement avancé, notamment avant chimiothérapie néo-adjuvante, un scanner thoraco-abdominopelvien ou une échographie du creux axillaire peuvent apporter des informations morphologiques complémentaires. Pour autant et à l'heure actuelle, le staging ganglionnaire axillaire relève toujours de la chirurgie du creux axillaire (biopsie du ganglion sentinelle ou curage) avec examen histologique, malgré la morbidité associée à ce geste $[4,5]$.

L'imagerie par tomographie par émission de positons au fluoro-déoxy-glucose marqué par le fluor-18 (TEP-FDG) a montré son intérêt pour déterminer l'atteinte ganglionnaire axillaire et extra-axillaire ainsi que l'existence de métastases à distance dans le staging initial des cancers du sein localement avancés [6,7]. Dans un certain nombre de cas, elle permet ainsi de modifier la prise en charge thérapeutique, notamment lors du choix des aires ganglionnaires locorégionales à irradier [8]. Elle apparaît également contributive dans l'évaluation précoce de la réponse à la chimiothérapie néo-adjuvante $[9,10]$. En revanche, son apport n'est pas démontré dans la prise en charge initiale des cancers diagnostiqués à un stade précoce, en raison de son manque de sensibilité sur l'atteinte axillaire et de la faible prévalence des métastases à distance.

L'objectif de notre travail a été d'évaluer de manière rétrospective les performances de la TEP-FDG chez des patientes porteuses d'un cancer du sein localement avancé et candidates à une chimiothérapie néo-adjuvante. En particulier, nous avons comparé les résultats du staging ganglionnaire axillaire obtenus par la TEP-FDG préthérapeutique à ceux de l'histologie du curage axillaire réalisé après la chimiothérapie néo-adjuvante.

\section{Patientes et méthodes}

\subsection{Patientes}

Parmi les patientes atteintes d'un cancer du sein explorées entre janvier 2004 et mai 2009 au centre Paul-Papin par TEP-FDG, nous avons inclus dans cette étude celles atteintes d'un cancer du sein unilatéral prouvé histologiquement dont le traitement a consisté en une chimiothérapie néo-adjuvante suivie d'une chirurgie mammaire et d'un curage ganglionnaire axillaire.

Les critères d'exclusion étaient les suivants : cancer du sein bilatéral, données manquantes sur le bilan d'extension ou sur l'histologie de la chirurgie mammaire et/ou axillaire, antécédent de néoplasie mammaire ou de tout autre cancer datant de moins de cinq ans.

Nous avons donc inclus finalement dans cette étude 89 patientes. Les principales caractéristiques de ces patientes sont résumées dans le Tableau 1.

\subsection{TEP-FDG}

L'examen a été réalisé après une période de jeûne d'au moins six heures. L'injection intraveineuse de fluoro-désoxyglucose marqué au fluor-18 $\left({ }^{18} \mathrm{~F}\right.$-FDG) a été faite dans le bras opposé au côté de la néoplasie mammaire après contrôle de la glycémie. Les patientes ont ensuite respecté 60 minutes de repos, au calme, en position allongée. Les images ont été obtenues sur une caméra TEP couplée à une tomodensitométrie (TDM) 8 barrettes Discovery ST General Electric GE-HC. Les données en émission ont été acquises en mode 2D $(5 \mathrm{MBq} / \mathrm{kg})$ jusque fin juillet 2008 puis en mode 3D (4 MBq/kg), du vertex à mi-cuisses, bras au-dessus de la tête, et reconstruites en un volume de coupes de 3,3 mm d'épaisseur. Ces données tomographiques ont été corrigées de l'atténuation par l'utilisation de la TDM.

Toutes les images ont été revues par le même médecin nucléaire sans connaissance du dossier des patientes. Comme décrit par Heusner et al. [11], les foyers hypermétaboliques ont été considérés comme positifs s'ils étaient d'intensité supérieure à la fixation normale et non compatibles avec une

Tableau 1

Caractéristiques de la population étudiée ( 89 patientes).

Characteristics of the population (89 patients).

\begin{tabular}{lrr}
\hline & Nombre & $\%$ \\
\hline Âge moyen : $50 \pm 12(26-79)$ & & \\
Sein & & 39 \\
$\quad$ Droit & 35 & 61 \\
Gauche & 54 & \\
Histologie & & 90 \\
Carcinome canalaire infiltrant & 80 & 9 \\
Carcinome lobulaire infiltrant & 8 & 1 \\
Tumeur sarcomatoïde néoplasique & 1 & 58 \\
Récepteurs & & 49 \\
Estrogènes positifs & 52 & 33 \\
Progestérone positif & 44 & 24 \\
Her2+ & 29 & \\
Triple négatif & 21 & 2 \\
Grade (SBR) & & 56 \\
I & 2 & 42 \\
II & 50 & 1 \\
III & 37 & \\
Non gradable & 1 & \\
\hline
\end{tabular}


fixation physiologique ou bénigne. Cette décision a été fondée sur l'expérience du médecin. L'analyse des données scanographiques permettait d'aider à la décision (taille, forme et type de la lésion en TDM), mais ne permettait pas à elle seule de positiver une lésion.

Une région d'intérêt a été déterminée manuellement sur le foyer hypermétabolique correspondant à la tumeur primitive et le pixel d'intensité maximum a été relevé en utilisant la Standardized Uptake Value (SUV). La SUVmax a été obtenue à partir de la formule suivante :

\section{SUVmax $=[$ concentration d'activité dans la \\ région d'intérêt $(\mathrm{MBq} / \mathrm{mL}) /$ dose injectée \\ $(\mathrm{MBq})] /$ poids du patient $(\mathrm{g})$}

Un foyer hypermétabolique ganglionnaire ou viscéral a été considéré comme pathologique selon le même critère visuel d'interprétation et sa SUVmax a été relevée. En cas de présence de plusieurs foyers hypermétaboliques ganglionnaires dans le même secteur (axillaire, rétropectoral, sus-claviculaire ou mammaire interne), le nombre de foyers et la SUVmax du foyer le plus intense ont été notés. En cas de foyer d'interprétation douteuse (difficultés à classer le foyer comme positif ou négatif), une décision consensuelle a été prise avec deux autres médecins nucléaires du centre.

Pour l'analyse des images TDM, la fenêtre de lecture a été adaptée en fonction de l'organe étudié (fenêtres osseuse, hépatique, pulmonaire).

\subsection{Bilan clinique et paraclinique}

Les données cliniques (palpation de la tumeur et des aires ganglionnaires, inflammation de l'étui cutané mammaire, suspicion de métastases à distance) ont été recueillies d'après le compte rendu de consultation préthérapeutique.

Les patientes ont bénéficié du bilan d'extension standard recommandé : radiographie thoracique, échographie hépatique et scintigraphie osseuse (caméra INFINIA, GE). Pour cinq d'entre elles, la radiographie thoracique et l'échographie hépatique ont été remplacées par un scanner thoraco-abdominopelvien. Les résultats de ces examens ont été repris par lecture des comptes rendus. En cas de suspicion de métastase à distance sur l'examen TEP-FDG, le résultat a été confronté à celui des examens additionnels réalisés pour confirmer le diagnostic (TDM diagnostique, IRM). Une patiente a été considérée comme métastatique si le bilan d'extension standard ou la TEP-FDG était en faveur d'une lésion secondaire (fixation intense sur un site métastatique classique en TEP-FDG et/ou examen du bilan standard interprété comme tel). En cas de doute sur ce bilan initial, la patiente était considérée comme métastatique si les examens additionnels réalisés confirmaient cette suspicion (examen d'imagerie complémentaire, biopsie).

Au sein de cette population, nous avons individualisé six patientes pour lesquelles une cytoponction ganglionnaire axillaire avait été réalisée lors du bilan initial avant l'examen TEP-FDG.

\subsection{Résultats histologiques de la chirurgie mammaire et du curage ganglionnaire axillaire}

Au décours de la chimiothérapie néo-adjuvante, les patientes ont bénéficié soit d'un traitement chirurgical mammaire conservateur $(n=41)$, soit d'une mastectomie $(n=48)$, associé à un curage ganglionnaire axillaire. L'étude histologique de la réponse à la chimiothérapie a été évaluée en utilisant la classification de Sataloff (Annexe 1) [12]. L'histologie du curage axillaire a constitué le gold standard pour définir le statut ganglionnaire axillaire initial des patientes et a été considérée comme positive si l'examen anatomopathologique montrait au moins une métastase ganglionnaire active et/ou des signes d'effet thérapeutique (NA, NC ou ND selon la classification de Sataloff). Dans les autres cas, l'histologie a été considérée comme négative (NB selon la classification de Sataloff) (Annexe 1).

\subsection{Analyse statistique}

Les variables quantitatives ont été présentées avec leur valeur moyenne \pm écart-type (valeurs extrêmes). Un test de concordance (coefficient kappa de Cohen) a été réalisé pour mesurer la corrélation entre les résultats obtenus en TEP-FDG sur l'atteinte ganglionnaire axillaire et le résultat histologique du curage (considéré comme le gold standard).

\section{Résultats}

\subsection{Tumeur primitive mammaire}

La néoplasie mammaire a été palpée cliniquement chez 87 patientes $(97,8 \%)$ et retrouvée en TEP-FDG chez 85 patientes $(95,5 \%)(\operatorname{SUVmax}=10,5 \pm 7,7$ [2,1-42]). Les quatre faux-négatifs en TEP-FDG correspondaient à deux carcinomes lobulaires infiltrants (CLI) de grades II et III (respectivement de 30 et $60 \mathrm{~mm}$ ), un carcinome canalaire infiltrant (CCI) de grade II de $30 \mathrm{~mm}$ et un CCI de grade II se présentant sous forme de microcalcifications diffuses.

L'histologie de la tumeur primitive a révélé 80 CCI (78 TEP positives, SUVmax = 10,6 \pm 7,6 [2,5-42]), huit CLI (6 TEP positives, $\operatorname{SUVmax}=6,6 \pm 4,7[2,1-13,9])$ et une tumeur sarcomatoïde néoplasique (TEP positive, SUVmax = 26,3).

L'examen TEP-FDG a mis de plus en évidence un hypermétabolisme de l'étui cutané chez dix des 17 patientes $(58,8 \%)$ présentant cliniquement un cancer du sein inflammatoire $(\mathrm{SUVmax}=3,0[2,3-5,2])$.

\subsection{Extension ganglionnaire locorégionale}

La présence d'adénopathie(s) axillaire(s) homolatérale(s) a été suspectée cliniquement chez 39 patientes (44\%). Au moins un foyer hypermétabolique ganglionnaire axillaire a été retrouvé en TEP-FDG chez 58 patientes (65\%) (SUVmax $=7,1 \pm 5,5[1,1-24,8]$ ), représentant un total de 223 foyers (moyenne par patiente $=3,9 \pm 3,2$ [1-14]).

La TEP-FDG a retrouvé des foyers hypermétaboliques ganglionnaires extra-axillaires, non soupçonnés par la clinique 
et le bilan d'imagerie conventionnelle, chez 25 patientes $(28 \%)$ : atteinte sus-claviculaire chez huit patientes (SUVmax $=6,9 \pm 7,1$ [2,5-22,4], 1 à 4 foyers), mammaire interne chez sept patientes (SUVmax $=2,4 \pm 0,6$ [1,5-3], 1 à 2 foyers), rétropectorale chez 21 patientes (SUVmax $=5,3 \pm 4,5 \quad[1,4-18,1], 1$ à 6 foyers). Aucune atteinte ganglionnaire (axillaire ou extra-axillaire) controlatérale à la tumeur primitive n'a été suspectée en TEP-FDG.

\subsection{Cytologie axillaire préthérapeutique}

Six patientes ont bénéficié d'une cytoponction ganglionnaire axillaire au moment du diagnostic. Dans tous les cas, l'atteinte axillaire était suspectée cliniquement et sur l'examen TEPFDG (SUVmax $=8,2 \pm 5,2[4,3-18,1]$ ). La cytologie a été positive à chaque fois. Lors du curage axillaire, cinq patientes ont été classées NA selon Sataloff (effet thérapeutique sans métastase active) et la sixième NB (pas d'effet thérapeutique ni de métastase active).

\subsection{Histologie du curage axillaire et TEP-FDG}

L'examen anatomopathologique du curage axillaire a montré des métastases ganglionnaires actives NC (effet thérapeutique, mais métastase axillaire toujours présente) ou ND (métastase axillaire toujours présente et viable et pas d'effet thérapeutique) chez 34 patientes (38,2\%), une réponse
Tableau 2

Analyse histologique du curage axillaire après chimiothérapie néo-adjuvante et TEP-FDG préthérapeutique.

Comparison between histology of axillary dissection after neoadjuvant chemotherapy and initial PET/CT.

\begin{tabular}{|c|c|c|c|c|}
\hline & \multicolumn{2}{|c|}{ Histologie positive } & \multicolumn{2}{|c|}{ Histologie négative } \\
\hline & $\begin{array}{l}\text { Sataloff } \\
\text { (NC et ND) }\end{array}$ & $\begin{array}{l}\text { Sataloff } \\
\text { (NA) }\end{array}$ & $\begin{array}{l}\text { Sataloff } \\
\text { (NB) }\end{array}$ & Total \\
\hline TEP positive & 27 & 20 & 11 & 58 \\
\hline TEP négative & 7 & 5 & 19 & 31 \\
\hline Total & 34 & 25 & 30 & 89 \\
\hline
\end{tabular}

NA : évidence d'un effet thérapeutique, pas de maladie résiduelle ; NB : pas de métastase ou d'effet thérapeutique ; NC : évidence d'un effet thérapeutique mais métastase axillaire toujours présente ; ND : métastase axillaire toujours présente et viable et pas d'effet secondaire.

NA chez 25 patientes $(28,1 \%)$ et un statut NB chez 30 patientes $(33,7 \%)$. Le Tableau 2 présente le tableau de contingence des résultats histologiques du curage axillaire (pris comme référence dans notre étude) et de la TEP-FDG. La valeur du coefficient kappa de concordance est de 0,43 $\pm 0,11(0,22-$ $0,63)$. Les sensibilité, spécificité, valeur prédictive positive et valeur prédictive négative de la TEP-FDG pour la détection d'un envahissement ganglionnaire axillaire sont ainsi calculées à, respectivement, $80 \%$ (47/59), $63 \%$ (19/30), $81 \%(47 / 58)$ et $61 \%(19 / 31)$.

Tableau 3

Présentation des cas discordants entre la TEP-FDG et l'histologie du curage axillaire.

Presentation of discordants cases between FDG-PET and histology of axillary dissection.

\begin{tabular}{|c|c|c|c|c|}
\hline \multicolumn{2}{|c|}{ Statut ganglionnaire axillaire } & \multicolumn{2}{|l|}{ Tumeur primitive } & \multirow{2}{*}{$\begin{array}{l}\text { Atteinte extra-axillaire en TEP } \\
0\end{array}$} \\
\hline $\begin{array}{l}\text { TEP (nombre de } \\
\text { foyers ; SUVmax) }\end{array}$ & $\begin{array}{l}\text { Histologie (Sataloff, nombre } \\
\text { de métastases actives/ } \\
\text { ganglions prélevés) }\end{array}$ & Histologie & TEP (SUVmax) & \\
\hline Négative & Positive (NA, 0/12) & CCI grade III & Positive $(10,3)$ & 0 \\
\hline Négative & Positive (ND, 2/6) & CCI grade II & Positive $(3,9)$ & 0 \\
\hline Négative & Positive (ND, 1/12) & CCI grade II & Positive $(6,7)$ & 0 \\
\hline Négative & Positive (NA, 0/18) & CCI grade II & Négative & 0 \\
\hline Négative & Positive (NC, 1/26) & CCI grade II & Positive $(6,0)$ & 0 \\
\hline Négative & Positive (NC ou D, 6/12) & CCI grade II & Positive $(5,6)$ & 0 \\
\hline Négative & Positive (NC, 1/9) & CCI grade II & Positive $(7,9)$ & 0 \\
\hline Négative & Positive (NA, 0/15) & CCI grade III & Positive $(5,1)$ & 0 \\
\hline Négative & Positive (NA, 0/8) & CCI grade II & Négative & 0 \\
\hline Négative & Positive (NA, 0/18) & CCI grade III & Positive $(12,3)$ & 0 \\
\hline Négative & Positive (ND, 1/8) & CCI bifocal (II et III) & Positive $(11,9$ et 7,7$)$ & Mammaire interne $(n=2$, SUVmax $=2,6)$ \\
\hline Négative & Positive (ND, 1/16) & CCI grade II & Positive $(11,8)$ & 0 \\
\hline Positive $(5 ; 3,5)$ & Négative $(\mathrm{NB}, 0 / 18)$ & CCI grade II & Positive $(10,9)$ & 0 \\
\hline Positive $(3 ; 11,1)$ & Négative $(\mathrm{NB}, 0 / 29)$ & CCI grade II & Positive (19) & 0 \\
\hline Positive $(1 ; 5,1)$ & Négative $(\mathrm{NB}, 0 / 20)$ & CCI grade II & Positive $(9,3)$ & Mammaire interne $(n=1, \operatorname{SUVmax}=1,6)$ \\
\hline Positive $(6 ; 5,2)$ & Négative $(\mathrm{NB}, 0 / 14)$ & CCI grade II & Positive $(5,3)$ & Rétropectoral $(n=2$, SUVmax $=2,0)$ \\
\hline Positive $(1 ; 7,2)$ & Négative $(\mathrm{NB}, 0 / 13)$ & CCI grade II & Positive (21) & 0 \\
\hline Positive $(4 ; 9,3)$ & Négative $(\mathrm{NB}, 0 / 20)$ & CCI grade II & Positive $(10,3)$ & 0 \\
\hline Positive $(1 ; 1,1)$ & Négative $(\mathrm{NB}, 0 / 8)$ & CCI grade III & Positive $(22,7)$ & Mammaire interne $(n=2$, SUVmax $=3,0)$ \\
\hline Positive $(4 ; 2,4)$ & Négative $(\mathrm{NB}, 0 / 14)$ & CCI grade III & Positive $(12,9)$ & 0 \\
\hline Positive $(2 ; 1,4)$ & Négative $(\mathrm{NB}, 0 / 11)$ & CCI grade III & Positive $(5,5)$ & 0 \\
\hline Positive $(2 ; 1,6)$ & Négative $(\mathrm{NB}, 0 / 18)$ & CLI grade III & Négative & 0 \\
\hline Positive $(1 ; 1,3)$ & Négative $(\mathrm{NB}, 0 / 16)$ & CCI grade III & Positive $(19,3)$ & 0 \\
\hline
\end{tabular}

CCI : carcinome canalaire infiltrant ; NA : évidence d'un effet thérapeutique, pas de maladie résiduelle ; NB : pas de métastase ou d'effet thérapeutique ; NC : évidence d'un effet thérapeutique mais métastase axillaire toujours présente ; ND : métastase axillaire toujours présente et viable et pas d'effet secondaire. 
Les cas discordants entre l'histologie et la TEP-FDG sont répertoriés dans le Tableau 3. Douze patientes présentaient une histologie positive du curage axillaire post-chimiothérapie alors que la TEP-FDG initiale était négative. À l'inverse, l'histologie était négative chez 11 patientes dont la TEP-FDG initiale était positive (SUVmax axillaire $=4,5 \pm 3,5 \quad[1,1-$ $11,1]$; nombre de foyers $=2,7 \pm 1,8[1-6])$.

\subsection{Extension à distance}

Cliniquement, aucune patiente n'était suspecte d'être métastatique. À l'issue du bilan, cinq patientes $(5,6 \%)$ ont été considérées comme métastatiques : osseux $(n=4)$, hépatique $(n=1)$, pulmonaire $(n=1)$.

$\mathrm{Au}$ niveau osseux, la scintigraphie osseuse était positive chez deux patientes et la TEP-FDG chez quatre patientes (avec confirmation IRM dans les deux cas discordants avec la scintigraphie osseuse). Sur ces quatre patientes, on notait la présence de lésions lytiques sur les coupes TDM chez trois d'entre elles et l'absence de lésion TDM pour la dernière. Dans trois autres cas, la scintigraphie était douteuse mais la TEPFDG et l'IRM étaient négatives.

Au niveau hépatique, l'échographie et la TEP-FDG étaient concordantes chez une patiente pour attester d'une atteinte secondaire (une lésion avec SUVmax à 5,6 en TEP). Chez deux patientes, la TEP-FDG était positive avec, dans un cas, une lésion hypermétabolique (SUVmax à 4,0) non confirmée par échographie et IRM et, dans l'autre cas, un foie très hétérogène avec au moins deux foyers hypermétaboliques suspects dont l'un en regard d'une lésion hypodense au recalage TDM (SUVmax à 5,3). Pour cette dernière patiente, l'IRM n'a été réalisée qu'après chimiothérapie et n'a pas mis en évidence de lésion suspecte (TEP-FDG de contrôle concomitante en faveur d'une réponse complète) (Fig. 1).

Pour une autre patiente, l'échographie et la TEP-FDG (une lésion avec SUVmax à 6,2) étaient considérées comme positives, mais l'IRM s'est révélée négative suspectant une adénomatose, et la biopsie a confirmé l'adénomatose hépatique.
Aucune atteinte pleuropulmonaire n'a été suspectée sur la radiographie standard. La TEP-FDG a évoqué chez une patiente des lésions secondaires pulmonaires bilatérales (opacités disséminées dans les deux champs pulmonaires, SUVmax $=10,0$ ) avec confirmation sur un scanner thoracique diagnostique.

\section{Discussion}

Dans notre série, la TEP-FDG a détecté la néoplasie mammaire primitive dans 95,5\% des cas, avec davantage de faux-négatifs pour les carcinomes lobulaires (2/8, soit $25 \%$ ) que pour les canalaires infiltrants $(2 / 80$, soit $2,5 \%)$, ce qui est concordant avec les résultats décrits dans la littérature [13,14].

L'examen TEP-FDG initial a suspecté une atteinte ganglionnaire axillaire chez $65 \%$ des patientes, lui conférant sur les données histologiques obtenues après traitement une sensibilité de $80 \%$, une valeur prédictive positive de $81 \%$ et une spécificité de $63 \%$. Dans une méta-analyse de 2009 de Peare et al. [15], les valeurs de sensibilité tout stade confondu variaient de 20 à $100 \%$. Les principales raisons invoquées par l'auteur pour expliquer ce manque de sensibilité étaient, d'une part, l'incapacité de la TEP-FDG à détecter les micrométastases et, d'autre part, le fait que l'intensité de fixation ganglionnaire soit corrélée à celle de la tumeur primitive [15]. Les limites de la TEP-FDG pour le staging ganglionnaire axillaire dans les tumeurs de petite taille sont ainsi bien connues, une métaanalyse récente obtenant une sensibilité de $53 \%$ pour les tumeurs de stade T1 et de $67 \%$ pour les tumeurs de stade T2 $[16,17]$.

La spécificité de la TEP-FDG dans notre étude a été de $63 \%$. La méta-analyse de Peare [15] a montré des valeurs de spécificité variant de 66 à $100 \%$ lorsque le curage axillaire a été réalisé au décours immédiat de la TEP-FDG. La spécificité plus faible dans notre étude peut s'expliquer tout d'abord par la comparaison de données TEP-FDG préthérapeutiques et de données histologiques obtenues quelques mois plus tard en fin de chimiothérapie néo-adjuvante. Une hypothèse envisageable

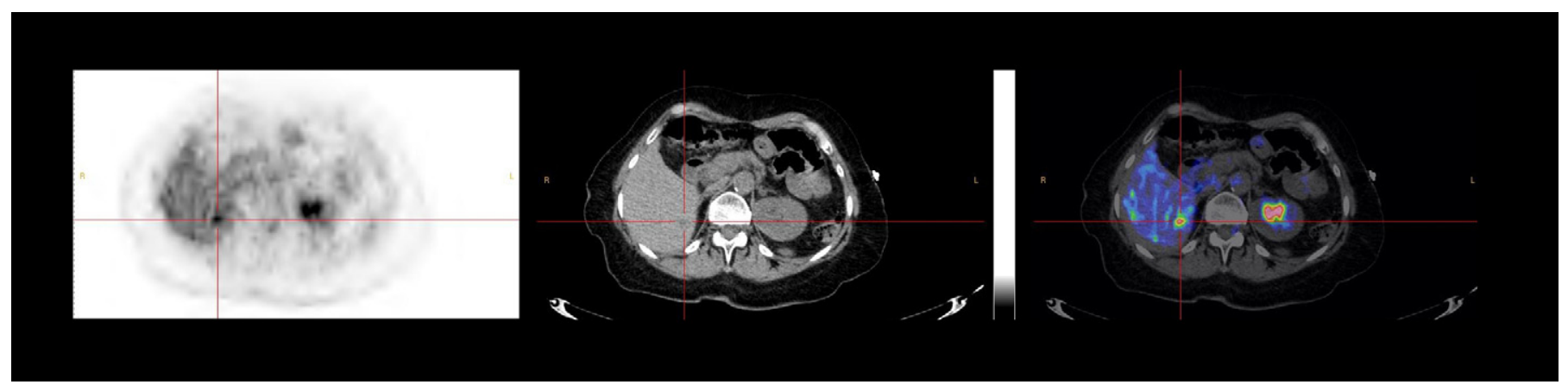

Fig. 1. Patiente de 68 ans présentant un carcinome canalaire infiltrant grade III, triple négatif. La TEP-FDG montre une fixation hépatique hétérogène avec individualisation d'au moins deux foyers intenses, dont l'un correspond à une lésion arrondie hypodense sur le scanner de repérage. L'échographie préthérapeutique n'identifiait pas de lésion suspecte. L'IRM hépatique a été réalisée après la chimiothérapie.

Sixty-eight years old patient with an infiltrating ductal carcinoma grade III, triple negative. FDG-PET shows a heterogeneous liver metabolism. We can individualize at least two intense uptakes, one of which corresponds to a hypodense lesion on CT. Pretherapeutic ultrasonography showed no suspect lesion. Hepatic MRI was performed after chemotherapy. 




Fig. 2. Patiente de 37 ans présentant un carcinome canalaire infiltrant grade II, RH-, Her2+. La TEP-FDG initiale montre un foyer hypermétabolique (SUVmax à $5,2)$ en regard d'un ganglion axillaire homolatéral. Une cytoponction préthérapeutique avait mis en évidence un envahissement métastatique axillaire. L'histologie du curage axillaire réalisé après chimiothérapie néo-adjuvante se révèle négative, NB selon la classification de Sataloff.

Thirty-seven years old patient with an infiltrating ductal carcinoma grade III, hormone receptors negative, Her 2 positive. Initial FDG-PET shows an uptake $($ SUVmax $=5.2)$ in an ipsilateral axillary lymph node. Fine needle aspiration performed before the beginning of the treatment had revealed a metastatic axillary involvement. Histology of axillary dissection performed after neoadjuvant chemotherapy proved negative, NB Sataloff's classification.

est que la chimiothérapie puisse convertir un ganglion envahi en un ganglion histologiquement négatif. Nous avons pu noter, en effet, le cas d'une patiente dont la cytoponction axillaire initiale a mis en évidence un envahissement ganglionnaire et dont l'analyse du curage après traitement ne montrait aucun signe d'effet thérapeutique ni métastase active (NA dans la classification de Sataloff), conduisant de ce fait à considérer cette patiente comme un faux-positif TEP-FDG (Fig. 2). Alberini et al. [8] ont également décrit dans leur série de cancers du sein inflammatoires deux cas de faux-positifs TEP, classés NB selon la classification de Sataloff. Nous avons donc probablement sous-estimé la spécificité de la TEP-FDG et pointons les lacunes du bilan standard sur le staging ganglionnaire quand il ne comprend pas d'échographie couplée à une cytoponction, notamment chez les patientes à haut risque d'atteinte ganglionnaire (dans notre série, une cytoponction n'a été réalisée que chez six patientes).

Par ailleurs, nous avions retenu comme positives en TEPFDG les fixations ganglionnaires supérieures au bruit de fond local. Dans la littérature, des auteurs ont privilégié des méthodes quantitatives (valeurs de SUVmax supérieures à 2,5 [7], ratios lésion/bruit de fond [18], couplés éventuellement à une correction de l'effet de volume partiel [10]), sans qu'aucun seuil de positivité ne puisse être défini. L'avantage de l'interprétation visuelle est sa relative facilité de mise en œuvre, mais elle peut majorer le taux de faux-positifs d'origine inflammatoire, notamment dans les cas de fixation légèrement supérieure à celle du bruit de fond. Pour ces cas « litigieux », la

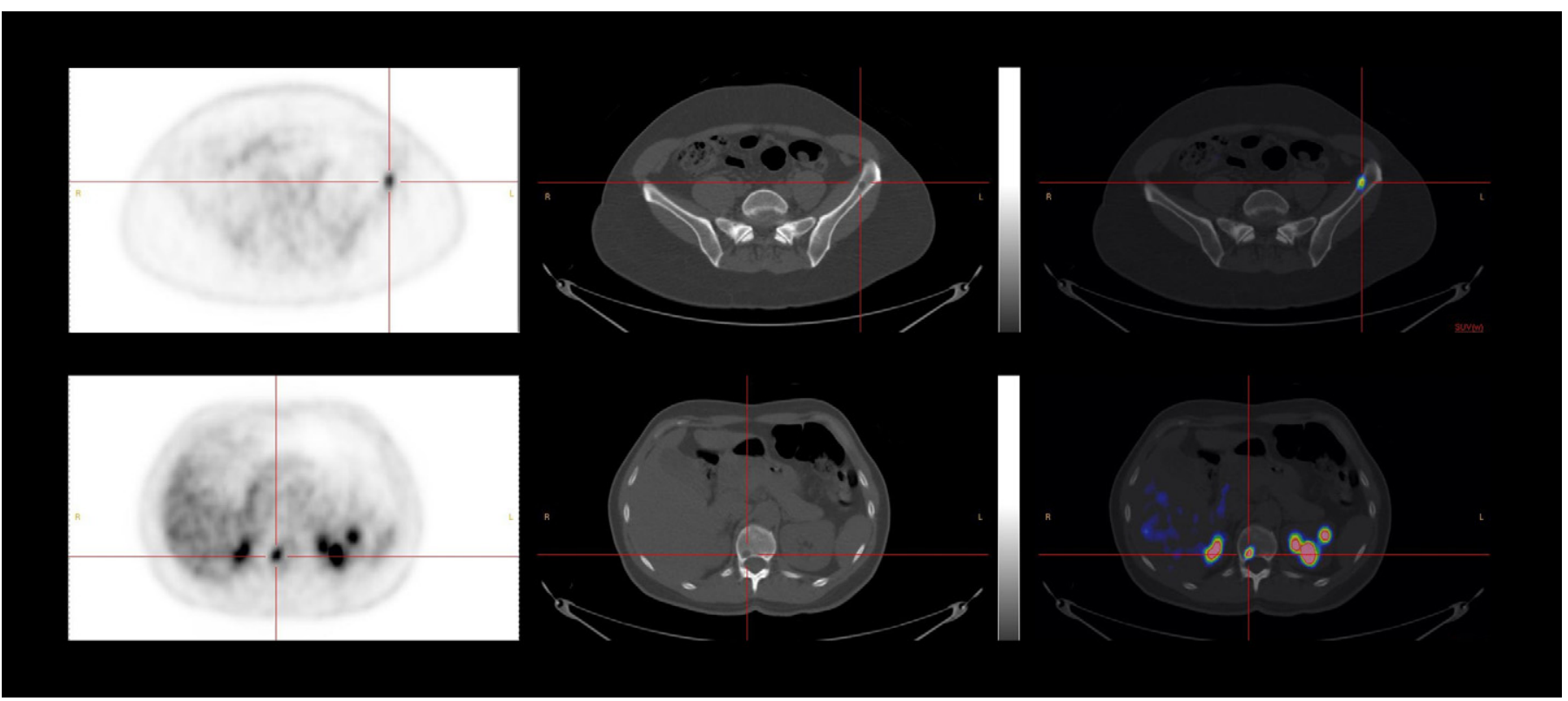

Fig. 3. Patiente de 47 ans présentant un carcinome canalaire infiltrant grade II, RH+, Her2-. La TEP-FDG initiale met en évidence des lésions osseuses lytiques hypermétaboliques du bassin (aile iliaque gauche) et du rachis (T12). La scintigraphie osseuse s'était révélée négative.

Forty-seven years old patient with an infiltrative ductal carcinoma grade II, hormone receptors positive, Her 2 negative. Initial FDG-PET reveals hypermetabolic lytic bone lesions of the pelvis (left iliac wing) and spine (T12). Bone scan had proved negative. 
réalisation d'une cytoponction échoguidée du ganglion hypermétabolique pourrait être proposée afin de définir le statut axillaire avant traitement.

L'extension ganglionnaire extra-axillaire a été observée dans $16 \%$ des cas (6/38) par Straver et al. [18] et dans $28 \%$ des cas (17/60) par Aukema et al. [19]. L'atteinte mammaire interne ou sous-claviculaire constitue des facteurs de mauvais pronostic reconnus [20,21]. Des études ont démontré la supériorité de la TEP-FDG pour sa détection par rapport au bilan conventionnel, notamment sur la chaîne mammaire interne $[19,22,23]$; la région sous-claviculaire pouvant être explorée plus aisément par échograhie.

Nous avons constaté dans notre étude une atteinte ganglionnaire extra-axillaire chez 25 patientes $(28 \%)$. Aucune n'était suspectée par le bilan d'extension standard et il n'y a pas eu de confirmation histologique ou par une autre modalité d'imagerie. Pour autant, la forte spécificité de la TEP-FDG est reconnue et incite à prendre en compte les résultats de la TEP pour adapter le traitement du cancer [6,7], en particulier lors du choix des aires ganglionnaires locorégionales à irradier. L'apport de la TEP-FDG induit un changement majeur dans l'attitude thérapeutique initialement prévue dans $15,6 \%$ des cas (18/115) dans l'étude récente de Garami et al. [24].

Concernant la présence de métastases à distance, nous avons considéré dans cette série $5,6 \%$ (5/89) de patientes métastatiques, valeur comparable à celles de van der Hoeven et al. [25] (8\% sur une population de 48 patientes), Schirrmeister et al. [26] (6 \% sur 117 patientes) ou Weir et al. [27] (5\% sur 84 patientes). L'étude de Koolen et al. [28] a démontré la supériorité de la TEP-FDG sur le bilan conventionnel pour la recherche de lésions à distance dans les cancers du sein localement avancés avec une sensibilité et une valeur prédictive négative de $100 \%$. La TEP-FDG a diagnostiqué deux atteintes osseuses manquées par la scintigraphie osseuse (Fig. 3). L'os est décrit comme le site le plus fréquent de lésions non documentées par le bilan standard. Des études prospectives ont en effet révélé en TEPFDG des patientes métastatiques ignorées par la scintigraphie osseuse : six sur 60, soit $10 \%$, dans l'étude de Fuster et al. de 2008 [7], trois sur 39, soit 7,7 \%, dans l'étude de Groheux et al. de 2008 [29] et quatre sur 131, soit $3 \%$, des cas dans l'étude de 2011 de cette même équipe [30].

Dans notre série, pour l'atteinte hépatique, nous avons eu un cas de faux-positif TEP-FDG correspondant à un adénome hépatique à l'histologie. Cette situation est exceptionnelle, mais il a déjà été rapporté un cas identique dans un suivi de cancer du sein [31].

\section{Conclusion}

En conclusion, cette étude rétrospective confirme l'intérêt de l'examen TEP-FDG dans le staging initial des cancers du sein avant chimiothérapie néo-adjuvante. La TEP a ainsi suggéré l'existence d'une atteinte ganglionnaire extra-axillaire méconnue chez $28 \%$ des patientes, cette information pouvant modifier le choix des aires ganglionnaires à irradier et le schéma thérapeutique global. Par ailleurs, une positivité de la
TEP au niveau ganglionnaire axillaire est très évocatrice d'une atteinte métastatique. Néanmoins, les cas d'interprétation douteuse devraient conduire à la réalisation d'une cytoponction échoguidée du ganglion hypermétabolique afin d'optimiser le staging préthérapeutique.

\section{Déclaration d'intérêts}

Les auteurs déclarent ne pas avoir de conflits d'intérêts en relation avec cet article.

\section{Annexe 1. Classification de Sataloff/Sataloff's classification.}

Tumeur primaire mammaire :

- TA : effet thérapeutique total ou presque total ;

- TB : effet thérapeutique de plus de $50 \%$ mais pas total ;

- TC : moins de $50 \%$ d'effet thérapeutique ;

- TD : pas d'effet thérapeutique.

Ganglions axillaires :

- NA : évidence d'un effet thérapeutique, pas de maladie résiduelle ;

- NB : pas de métastase ou d'effet thérapeutique ;

- NC : évidence d'un effet thérapeutique mais métastase axillaire toujours présente ;

- ND : métastase axillaire toujours présente et viable et pas d'effet thérapeutique.

\section{Références}

[1] Horner MJRL, Krapcho M, et al. (eds). SEER Cancer Statistics Review, 1975-2006. MD, USA: National Cancer Institute Bethesda; 2006.

[2] ${ }^{\mathbb{C}}$ La situation du cancer en France en 2010. http://www.e-cancer.fr.

[3] van Deurzen CH, Vriens BE, Tjan-Heijnen VC, van der Wall E, Albregts $\mathrm{M}$, van Hilligersberg R, et al. Accuracy of sentinel node biopsy after neoadjuvant chemotherapy in breast cancer patients: a systematic review. Eur J Cancer 2009;45:3124-30.

[4] Blanchard DK, Donohue JH, Reynolds C, Grant CS. Relapse and morbidity in patients undergoing sentinel lymph node biopsy alone or with axillary dissection for breast cancer. Arch Surg 2003;138:482-7 [discussion 7-8].

[5] Mansel RE, Fallowfield L, Kissin M, Goyal A, Newcombe RG, Dixon JM, et al. Randomized multicenter trial of sentinel node biopsy versus standard axillary treatment in operable breast cancer: the ALMANAC Trial. J Natl Cancer Inst 2006;98:599-609.

[6] Cermik TF, Mavi A, Basu S, Alavi A. Impact of FDG PET on the preoperative staging of newly diagnosed breast cancer. Eur J Nucl Med Mol Imaging 2008;35:475-83.

[7] Fuster D, Duch J, Paredes P, Velasco M, Munoz M, Santamaria G, et al. Preoperative staging of large primary breast cancer with [18F]fluorodeoxyglucose positron emission tomography/computed tomography compared with conventional imaging procedures. J Clin Oncol 2008;26:474651.

[8] Alberini JL, Lerebours F, Wartski M, Fourme E, Le Stanc E, Gontier E, et al. 18F-fluorodeoxyglucose positron emission tomography/computed tomography (FDG-PET/CT) imaging in the staging and prognosis of inflammatory breast cancer. Cancer 2009;115:5038-47. 
[9] Brunotte F, Cochet A, Toubeau M, Dygai-Cochet I, Riedinger JM. Place de l'imagerie dans l'évaluation de l'efficacité des traitements dans le cancer du sein. Med Nucl Imagerie Fonctionnelle Metabol 2010;34:5865.

[10] Rousseau C, Devillers A, Campone M, Campion L, Ferrer L, Sagan C, et al. FDG PET evaluation of early axillary lymph node response to neoadjuvant chemotherapy in stage II and III breast cancer patients. Eur J Nucl Med Mol Imaging 2011;38:1029-36.

[11] Heusner TA, Kuemmel S, Hahn S, Koeninger A, Otterbach F, Hamami $\mathrm{ME}$, et al. Diagnostic value of full-dose FDG PET/CT for axillary lymph node staging in breast cancer patients. Eur J Nucl Med Mol Imaging 2009;36:1543-50.

[12] Sataloff DM, Mason BA, Prestipino AJ, Seinige UL, Lieber CP, Baloch Z. Pathologic response to induction chemotherapy in locally advanced carcinoma of the breast: a determinant of outcome. J Am Coll Surg 1995;180:297-306.

[13] Eubank WB, Mankoff DA. Evolving role of positron emission tomography in breast cancer imaging. Semin Nucl Med 2005;35:84-99.

[14] Hodgson NC, Gulenchyn KY. Is there a role for positron emission tomography in breast cancer staging? J Clin Oncol 2008;26:712-20.

[15] Peare R, Staff RT, Heys SD. The use of FDG-PET in assessing axillary lymph node status in breast cancer: a systematic review and meta-analysis of the literature. Breast Cancer Res Treat 2010;123:281-90.

[16] Cooper KL, Harnan S, Meng Y, Ward SE, Fitzgerald P, Papaioannou D, et al. Positron emission tomography (PET) for assessment of axillary lymph node status in early breast cancer: a systematic review and metaanalysis. Eur J Surg Oncol 2011;37:187-98.

[17] Veronesi U, De Cicco C, Galimberti VE, Fernandez JR, Rotmensz N, Viale G, et al. A comparative study on the value of FDG-PET and sentinel node biopsy to identify occult axillary metastases. Ann Oncol 2007; 18:473-8.

[18] Straver ME, Aukema TS, Olmos RA, Rutgers EJ, Gilhuijs KG, Schot ME, et al. Feasibility of FDG PET/CT to monitor the response of axillary lymph node metastases to neoadjuvant chemotherapy in breast cancer patients. Eur J Nucl Med Mol Imaging 2010;37:1069-76.

[19] Aukema TS, Straver ME, Peeters MJ, Russell NS, Gilhuijs KG, Vogel WV, et al. Detection of extra-axillary lymph node involvement with FDG PET/ CT in patients with stage II-III breast cancer. Eur J Cancer 2010;46:3205-10.

[20] Newman LA, Kuerer HM, Fornage B, Mirza N, Hunt KK, Ross MI, et al. Adverse prognostic significance of infraclavicular lymph nodes detected by ultrasonography in patients with locally advanced breast cancer. Am J Surg 2001;181:313-8.

[21] Sugg SL, Ferguson DJ, Posner MC, Heimann R. Should internal mammary nodes be sampled in the sentinel lymph node era? Ann Surg Oncol 2000;7:188-92.

[22] Danforth Jr DN, Aloj L, Carrasquillo JA, Bacharach SL, Chow C, Zujewski $\mathrm{J}$, et al. The role of 18F-FDG-PET in the local/regional evaluation of women with breast cancer. Breast Cancer Res Treat 2002;75:135-46.

[23] Eubank WB, Mankoff DA, Takasugi J, Vesselle H, Eary JF, Shanley TJ, et al. 18fluorodeoxyglucose positron emission tomography to detect mediastinal or internal mammary metastases in breast cancer. J Clin Oncol 2001;19:3516-23.

[24] Garami Z, Hascsi Z, Varga J, Dinya T, Tanyi M, Garai I, et al. The value of 18-FDG PET/CT in early-stage breast cancer compared to traditional diagnostic modalities with an emphasis on changes in disease stage designation and treatment plan. Eur J Surg Oncol 2011.

[25] van der Hoeven JJ, Krak NC, Hoekstra OS, Comans EF, Boom RP, van Geldere D, et al. 18F-2-fluoro-2-deoxy-d-glucose positron emission tomography in staging of locally advanced breast cancer. J Clin Oncol 2004;22:1253-9.

[26] Schirrmeister H, Kuhn T, Guhlmann A, Santjohanser C, Horster T, Nussle $\mathrm{K}$, et al. Fluorine-18 2-deoxy-2-fluoro-D-glucose PET in the preoperative staging of breast cancer: comparison with the standard staging procedures. Eur J Nucl Med 2001;28:351-8.

[27] Weir L, Worsley D, Bernstein V. The value of FDG positron emission tomography in the management of patients with breast cancer. Breast $\mathbf{J}$ 2005;11:204-9.

[28] Koolen BB, Vegt E, Rutgers EJ, Vogel WV, Stokkel MP, Hoefnagel CA, et al. FDG-avid sclerotic bone metastases in breast cancer patients: a PET/ CT case series. Ann Nucl Med 2011.

[29] Groheux D, Moretti JL, Baillet G, Espie M, Giacchetti S, Hindie E, et al. Effect of (18)F-FDG PET/CT imaging in patients with clinical Stage II and III breast cancer. Int J Radiat Oncol Biol Phys 2008;71:695-704.

[30] Groheux D, Giacchetti S, Espie M, Vercellino L, Hamy AS, Delord M, et al. The yield of 18F-FDG PET/CT in patients with clinical stage IIA, IIB, or IIIA breast cancer: a prospective study. J Nucl Med 2011;52:152634.

[31] Patel PM, Alibazoglu H, Ali A, Fordham E, LaMonica G. 'False-positive' uptake of FDG in a hepatic adenoma. Clin Nucl Med 1997;22:490-1. 